\title{
A Case Study on Parikartika W.S.R Fissue in Ano
}

\author{
Dnyaneshwar K Jadhav* \\ Kaychikitsa department, Shri Dhanwantri Ayurved Medical College \& Research Centre, India
}

Received: September 04, 2017; Published: September 15, 2017

*Corresponding author: Dnyaneshwar K Jadhav, M.D Kaychikitsa (Ayurved), D.P.K.,D.A.Y., D.M.T., C.PSY., C.S.D, Assistant Professor, Kaychikitsa department, Shri Dhanwantri Ayurved Medical College \& Research Centre, Mathura, India, Tel: 8451834859; Email: dj85015@gmail.com

\begin{abstract}
Parikartika is one of the ano-rectal disease. Its references available in Bruhatrayees. The word Parikartika means Parikartanavatvedana around guda Pradesh. It is also having the symptoms like burning sensation, sometime swelling, bleeding from anus. In modern science it can be correlated to fissure in ano. Here a case Report of a 34 Years-Male Patient having history of Parikartika (fissure in ano) from last 5 years. He was suffering from Gudapradeshikartanvatvedana (Excruciating pain), Gudapradeshialpashoth (swelling at anal region), Gudapradeshidaha (burning sensation at anal region), Malavashtmbha (Constipation), Saraktamalapravrutti (Stools streaked with blood) since last 10 days. Generally he got relief from Modern medicines, but this time medicines not give relief. He was treated with Ayurvedic medicine, which give effective result within 3 days with Ayurvedic treatment.
\end{abstract}

Keywords: Parikartika; Fissure in ano; Ayurved

\section{Introduction}

In Ayurvedicsamhita this Parikartika is described in different place. In charakSamhita it describe as complication of VirechanaVyapad (therapeutic purgation) [1]. In Susruta Samhita it describe as Bastivhyapad [2]. Kashyapa mention it's as Garbhini Vyapad (Disease occurs in Pregnancy) [3].

Fissure-in-ano has become most common and painful condition in ano-rectal disease. It is commonly seen in young age peoples and pregnant women. It is a very painful because of injury to somatic nerve supply to the anal region. In modern science treatments includes analgesics, antibiotics, laxatives and ointment and anal dilatation, sphincterotomy, fissurectomy. Surgeries of Fissurein-ano are expensive and require long stay in hospital. All these procedures having its own complications.

In Ayurveda there are so many preparations and best surgery procedure also. The condition fissure-in-ano, commonly encountered in ano-rectal practice has similar location, pathology and clinical features of parikartika like anal pain, burning sensation at anal, constipation, stools streaked with blood etc [4].

\section{Case Report}

A 34 year old male patient came to us with chief compliant of -

A. Gudapradeshikartanvatvedana (Excruciating pain

B. Gudapradeshialpashoth (swelling at anal region)

C. Gudapradeshidaha (burning sensation at anal region)

D. Malavashtmbha (Constipation)

\section{E. Saraktamalapravrutti (Stools streaked with blood)}

Patient had above complaints since last 10 days. H/o - fissure: since last 5 years. No H/o any major surgery.

\section{History of Personal Illness}

The patient has history of fissure since last 5 years. He was taken modern medicine for that. The patient was normal before 10 days ago. From last 10 days, then patient had been suffering from Gudapradeshikartanvatvedana (Excruciating pain), Gudapradeshialpashoth(swelling atanalregion), Gudapradeshidaha (burning sensation at anal region), Malavashtmbha (Constipation), Saraktamalapravrutti (Stools streaked with blood).this time all symptoms unbearable and pain and burning sensation doesn't stop with modern medicines. For Ayurvedic Treatment he came to our Ayurved clinic.
A. $\quad$ Nadi (pulse) $=90 / \mathrm{min}$.
B. Mala $($ stool $)=$ Malavashmbha, SaRakta.
C. Mutra (urine) $=$ Prakrut
D. Jihwa (tounge) $=$ Saam .
E. Agni = Kshudhamandya.
F. Shabda $($ speech $)=$ prakrut $($ Normal $)$.
G. Sparsha $($ skin $)=$ prakrut
H. Druka (eyes) $=$ prakrut 
I. $\quad$ Akruti $=$ krusha.

J. Bala = Madhyama.

K. Raktachaapa (B.P) $=110 / 90 \mathrm{~mm} / \mathrm{Hg}$.

\section{Material and Method}

\section{Method}

Center of study: NakshatraAyurved clinic and Research Center, Mumbai, simple Random Single Case Study.

\section{Material}

(Table 1-4).

Table 1: Showing Internal Medicines used in Case Study.

\begin{tabular}{|c|c|c|c|c|}
\hline Sr.No & Dravya & Dose & Duration & Anupana \\
\hline 1 & TrifalaGugul & $250 \mathrm{mg}$ & 2 bid & Koshnajala \\
\hline 2 & GandhakRasyana & $330 \mathrm{mg}$ & 1 bid & Koshnajala \\
\hline 3 & Kamadudha Rasa & $150 \mathrm{mg}$ & 2 bid & Koshnajala \\
\hline 4 & Cap.Castrolax & $700 \mathrm{mg}$ & 2 bid & Koshnajala \\
\hline 5 & Jatyadi tail & $2.5 \mathrm{ml}$ & Twice in day & $\begin{array}{c}\text { For local } \\
\text { application }\end{array}$ \\
\hline
\end{tabular}

Table 2: Showing Procedure used in Case Study.

\begin{tabular}{|c|c|}
\hline \multicolumn{2}{|c|}{ Awagaha } \\
\hline $\begin{array}{c}\text { Trifala Choorna }+ \\
\text { Daruharidra choorna }+ \\
\text { Kanshi choorna }\end{array}$ & Twice in days \\
\hline
\end{tabular}

Table 3: Showing Mode of Actions of Dravyas.

\begin{tabular}{|c|c|}
\hline Dravya & Mode of action \\
\hline TrifalaGugul [6] & $\begin{array}{c}\text { Shothgha, malasarak, Tridoshnashk, } \\
\text { Prevent constipation, help for heeling } \\
\text { fissure. }\end{array}$ \\
\hline GandhakRasyana [7] & $\begin{array}{c}\text { Stimulates peristaltic moment of } \\
\text { intestine, makes stool smooth, decreased } \\
\text { burning sensation, purified occult blood } \\
\text { of fissure. }\end{array}$ \\
\hline Kamadudha rasa [8] & $\begin{array}{c}\text { Regulate digestion system, pittasahmak, } \\
\text { Raktastambhakkarya, }\end{array}$ \\
\hline Cap.castrolax & $\begin{array}{c}\text { Its contain 700 mg Ernada tail (castor } \\
\text { oil) which is Vatanashak, laxative, } \\
\text { lubricant to GI-Sysytem. }\end{array}$ \\
\hline Jatyadi tail [9] & Vranaropak, Vatshamak, Lubrication. \\
\hline Awagahan & $\begin{array}{c}\text { Shotaghan, Vatashamak, Heeling activity. } \\
\text { (trifala+daruharidra+kanshi) }\end{array}$ \\
\hline
\end{tabular}

Table 4: Showing Regression of Symptoms during Treatment.

\begin{tabular}{|c|c|c|c|c|c|}
\hline Sr.No & Symptoms & $\begin{array}{c}\mathbf{0}^{\text {th }} \\
\text { day }\end{array}$ & $\begin{array}{c}\mathbf{1}^{\text {st }} \\
\text { day }\end{array}$ & $\begin{array}{c}\mathbf{2}^{\text {nd }} \\
\text { days }\end{array}$ & $\begin{array}{c}\mathbf{3}^{\text {rd }} \\
\text { days }\end{array}$ \\
\hline 1 & $\begin{array}{c}\text { Gudapradeshikartanvatvedana } \\
\text { (Excruciating pain) }\end{array}$ & +++ & ++ & + & 0 \\
\hline 2 & $\begin{array}{c}\text { Gudapradeshialpashoth } \\
\text { (swelling at anal region) }\end{array}$ & ++ & + & + & 0 \\
\hline 3 & $\begin{array}{c}\text { Gudapradeshidaha (burning } \\
\text { sensation at anal region) }\end{array}$ & +++ & ++ & + & 0 \\
\hline 4 & Malavashtmbha (Constipation) & +++ & + & + & 0 \\
\hline 5 & $\begin{array}{c}\text { Saraktamalapravrutti (Stools } \\
\text { streaked with blood) }\end{array}$ & ++ & 0 & 0 & 0 \\
\hline
\end{tabular}

\section{Discussion}

\section{Hetu}

Ahar

A. Rukshanasevan(daily biscuit \& other bakery product consumption)

B. Ruksha-Amla-lawanaAhar(fast food and junk foodweekly).

C. Madhaypaan (Alcoholism)

D. Heavy food (sabudana-once in week)

E. Low intake of water.

Vihar
A. Prolong standing.
B. Jagarana (late night sleeping hour)

\section{Manasika Nidan}

Chinta, krodha causes vata vrudhi.

\section{Samparpti [5]}

Main cause of Parikartikais RukshaGuna of vata Dosha. Prolong standing, late night sleeping, alcohol consumption and dry diet \& low intake of water all of this cause vataparkop (due to increased RukhaGuna of vata Dosha) [6-9]. In this case study previously patient doesn't get relief from modern treatment, its may be due to resistance of medicine due to prolong consumption of same medicine (Figure 1).

Hetu sevan
(Ushan,Ruksha,VishtmbhiAahar)
Constipation due to Lifestyle cause(prolong seating, late night sleeping hour)
Straining during defecation
Defecation with pain, itching,cracks at anal,blood streak with stool
Parikartika (Fissure-in-ano)
Digure 1: Main cause of Parikartikais RukshaGuna of vata

\section{Pathya}

Patienthas advised to -
A. Increased liquid intake.
B. Fibres food in diet.
C. Buttermilk (with mire and badishop powder).
D. One TSF of cow Gruta before start the food.
E. Avoid late night sleep.
F. Seat for $5 \mathrm{~min}$ after 1 hour standing.

\section{Observations and Result}

Clinical examination of the patients show revealed regression of symptom on first day only, due to our Ayurvedic management. 


\section{Conclusion}

All said that Ayurved has slow result, but if we give proper treatment in specific condition than Ayurved show magical result. This case is one of best example of Magic of Ayurveda.

\section{References}

1. Kashinath shastri, Gorakhnath chaturvedi (1996) Charak Samhita $23^{\text {rd }}$ edn., Uttarardha, siddhi-stan, Adhaya no. 6, shloka no. 61-62, Chukhambha vishbahrati publication,Varanasi, pp. 1027.

2. Kavi raja ambika dasshastri (1997) Sushrut Samhita $11^{\text {th }}$ edn., Chikitsa stan, Adhaya no-34, Shloka no.16, Varanasi, : Chukhambha sanskruta sanstahan publication, Varanasi, pp.151.

3. Sharma hemraj (1995) Kashyapa Samhita, $4^{\text {th }}$ edn, Garbhinichikitsastan, Chukhmba publication, p. 65.
4. Bailys \& Loves Short Practice Of Surgery $24^{\text {th }}$ edn, pp. 1253,1254 .

5. Kulkarni parajakta, Parikartika : case study, int j res Ayurved pharma 6(4): 450

6. Brhamananda Tripathi (2011) Sharangadhar-Samhita Sharagdharacharya Rachita, Madhaymakhanda, chapter-7, Vataka kalpana, Varanasi, pp. 206.

7. Krushna Gopal Bhawan (2015) Rasatantrasaravasidha prayoga sangraha, Pratham Khanda, kharaliya Rasayan 81: 225-228.

8. Krushna Gopal Bhawan (2015) Rasatantrasaravasidha prayoga sangraha, Pratham Khanda, kharaliya Rasayan 80: 223-224.

9. Brhamananda Tripathi (2011) Sharangadhar-samhita, Sharagdharacharyarachita, Madhaymakhanda, chapter-9, Gruta-tail kalpana, Varanasi, pp. 242.

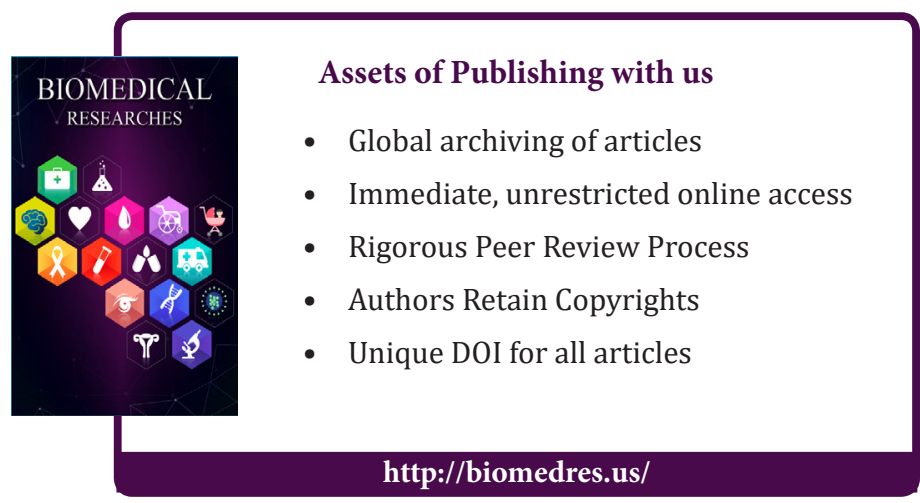

